\title{
Synthesis and properties of mixed-metal phosphido and phosphinidene clusters derived from reaction between $\mathrm{Ru}_{3}(\mathrm{CO})_{10}(\mu-\mathrm{H})\left(\mu-\mathrm{PPh}_{2}\right)$ and $\mathrm{Cp}^{\star} \mathrm{Mo}(\mathrm{CO})_{3} \mathrm{H}$
}

\author{
Jar-Chen Wang ${ }^{a}$, Yun Chi ${ }^{a}$, Shie-Ming Peng ${ }^{b}$, Gene-Hsiang Lee ${ }^{b}$, Shin-Guang Shyu ${ }^{c}$ \\ and Fung-Huang $\mathrm{Tu}^{\mathrm{a}}$ \\ ${ }^{a}$ Department of Chemistry, National Tsing Hua University, Hsinchu 30043 (China) \\ ${ }^{b}$ National Taiwan University, Taipei 10764 (Taiwan) \\ ${ }^{c}$ Institute of Chemistry, Academia Sinica, Taipei 11529 (Taiwan)
}

(Received October 27, 1993)

\begin{abstract}
The tetranuclear phosphido cluster $\mathrm{Cp}^{\star} \mathrm{MoRu}_{3}(\mathrm{CO})_{10}(\mu-\mathrm{H})_{2}\left(\mu-\mathrm{PPh}_{2}\right)(5)$ and the phosphinidene cluster $\mathrm{Cp}^{\star} \mathrm{MoRu} \mathrm{u}_{3}(\mathrm{CO})_{10}\left(\mu_{3}-\right.$ $\mathrm{H})\left(\mu_{3}-\mathrm{PPh}\right)(6)$ have been synthesized by reaction of $\mathrm{Ru}_{3}(\mathrm{CO})_{10}(\mu-\mathrm{H})\left(\mu-\mathrm{PPh}_{2}\right)$ with excess molybdenum hydride $\mathrm{Cp}{ }^{\star} \mathrm{Mo}(\mathrm{CO})_{3} \mathrm{H}$ in refluxing toluene solution. Crystals of 5 are monoclinic, space group $P 2$, with $a=10.708(2), b=10.848(4), c=14.885(2) \AA$, $\beta=97.63(2)^{r}, Z=2, R=0.021, R_{w}=0.019$ for 3025 observed reflections. The cluster has a tetrahedral array of metal atoms witl one edge-bridging and one face-bridging hydride, and a phosphido ligand bridged across a basal Ru-Ru edge. Thermolysis of 5 under similar conditions produced the phosphinidene cluster 6 in high yield via elimination of a benzene molecule, indicating that 5 is an intermediate in the formation of 6 . Subsequent treatment of 6 with $\mathrm{CO}$ caused fragmentation of the cluster affording a trinuclear phosphinidene cluster $\mathrm{Cp}^{\star} \mathrm{MoRu}_{2}(\mathrm{CO})_{8}(\mu-\mathrm{H})\left(\mu_{3}-\mathrm{PPh}\right)(7)$. Dimerization of 7 occurred upon thermolysis to afford hexanuclear $\mathrm{Cp}_{2}^{\star} \mathrm{Mo}_{2} \mathrm{Ru}_{4}(\mathrm{CO})_{12}(\mu-\mathrm{PPh})_{2}(8)$, which has also been characterized by X-ray diffraction. Crystal data of 8: space group $C 2 / c ; a=45.333(7), b=10.185(2), c=21.915(8) \AA, \beta=93.82(3)^{\circ}, Z=8$. The structure was solved by direct method and refined to $R$ and $R_{w}$ of 0.035 and 0.030 for 6135 observed reflections with $I>2 \sigma(I)$. The molecule adopts a square pyramidal $\mathrm{MoRu}_{4}$ skeleton with a tentacle $\mathrm{Mo}$ atom bridging a $\mathrm{Ru}-\mathrm{Ru}$ edge, on which the novel $\mu_{4}-\eta^{2}$-CO ligand is associated with the butterfly $\mathrm{Mo}_{2} \mathrm{Ru}_{2}$ array; one $\mu_{4}-\mathrm{PPh}$ ligand occupies a $\mathrm{MoRu}_{3}$ square face and a second $\mu_{3}-\mathrm{PPh}$ ligand caps a MoRu $\mathrm{Mace}_{2}$.
\end{abstract}

Key words: Rutherium; Molybdenum; Phosphido; Phosphinidene

\section{Introduction}

The chemistry of mixed-metal clusters of transitionmetals has been the subject of considerable research activity [1]. This has been stimulated by a belief that the various metals present in mixed-metal clusters may show reactivity patterns or structure very different from those of their homometallic analogues. With the aims of examining and comparing the basic of patterns of reactivity of heterometallic clusters, we have synthesized and characterized a series of trimetallic imido clusters $\mathrm{LWRu}_{2}(\mathrm{CO})_{8}(\mu-\mathrm{H})\left(\mu_{3}-\mathrm{NPh}\right), \mathrm{L}=\mathrm{Cp}$ and $\mathrm{Cp}^{\star}$ [2], and closely related phosphinidene clusters

Correspondence to: Dr. Y. Chi
$\mathrm{LWRu}_{2}(\mathrm{CO})_{8}(\mu-\mathrm{H})\left(\mu_{3}-\mathrm{PPh}\right)\left(\mathbf{1 a}, \mathrm{L}=\mathrm{Cp}, \mathbf{1 b}, \mathrm{L}=\mathrm{Cp}^{\star}\right)$ [3]. The reactivities of these imido and phosphinidene heterometallic clusters have been explored [4]. In contrast to the higher thermal stability of imido clusters, the phosphinidene derivative $1 \mathrm{~b}$, which was obtained from the direct treatment of $\mathrm{Ru}_{3}(\mathrm{CO})_{10}(\mu-\mathrm{H})\left(\mu-\mathrm{PPh}_{2}\right)$ with $\mathrm{Cp}$ * $\mathrm{W}(\mathrm{CO})_{3} \mathrm{H}$ through the formation of a tetranuclear precursor $\mathrm{Cp}^{\star} \mathrm{WRu}_{3}(\mathrm{CO})_{10}\left(\mu_{3}-\mathrm{H}\right)\left(\mu_{3}-\mathrm{PPh}\right)(2)$, can undergo dimerization by elimination of $\mathrm{H}_{2}$ and $\mathrm{CO}$ to afford two hexanuclear cluster derivatives (3) and (4) with an identical structural formula $\mathrm{Cp}_{2}^{\star} \mathrm{W}_{2}$ $\mathrm{Ru}_{4}(\mathrm{CO})_{12}(\mu-\mathrm{PPh})_{2}$ (Scheme 1) [5]. This variation in reactivity regarding the phosphinidene clusters is clearly due to the phosphorus atom being bigger than nitrogen, and the possibility of increased coordination capability [6]. 


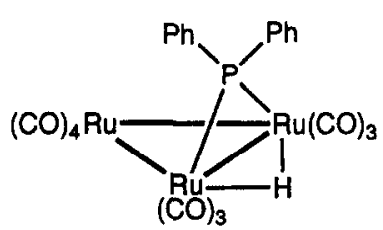

(i)
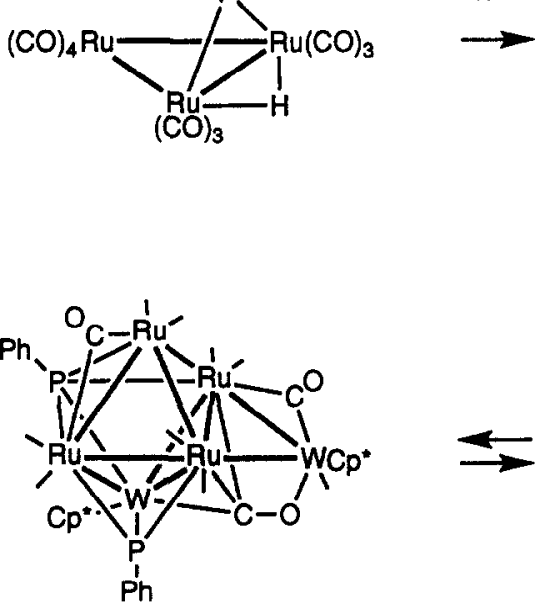

(4)

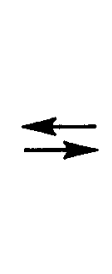

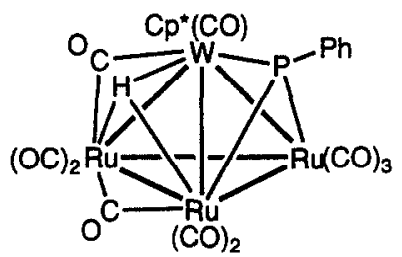

(2)

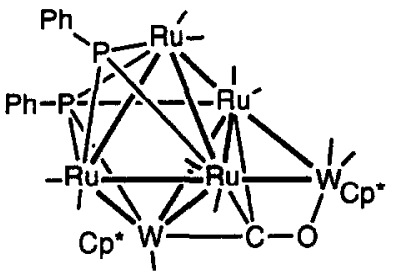

(3) (ii)

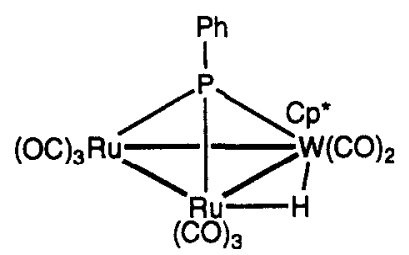

(1a)

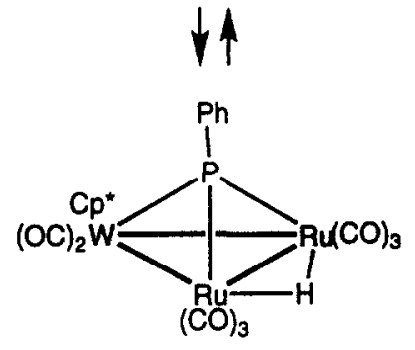

(1b)

Scheme 1.

In the present work on cluster assembly reactions, we have varied the transition-metal atoms in an attempt to examine the influence of transition metal atoms on the structure and reactivity of cluster compounds. We therefore reacted $\mathrm{Ru}_{3}(\mathrm{CO})_{10}(\mu-\mathrm{H})(\mu-$ $\mathrm{PPh}_{2}$ ) with $\mathrm{Cp}^{\star} \mathrm{Mo}(\mathrm{CO})_{3} \mathrm{H}$. From the reaction mixture, a small amount of an unknown phosphido cluster $\mathrm{Cp}^{\star} \mathrm{MoRu}_{3}(\mathrm{CO})_{10}(\mu-\mathrm{H})_{2}\left(\mu-\mathrm{PPh}_{2}\right)(5)$ was isolated, in addition to the expected tetranuclear phosphinidene cluster $\mathrm{Cp}^{\star} \mathrm{MoRu}_{3}(\mathrm{CO})_{10}\left(\mu_{3}-\mathrm{H}\right)\left(\mu_{3}-\mathrm{PPh}\right)(6)$ and trinuclear cluster $\mathrm{Cp}^{\star} \mathrm{MoRu}_{2}(\mathrm{CO})_{8}(\mu-\mathrm{H})\left(\mu_{3}-\mathrm{PPh}\right) \quad$ (7); their relationships were established. Furthermore, heating of $\mathbf{7}$ in toluene afforded only one hexanuclear derivative $\mathrm{Cp}_{2}^{\star} \mathrm{Mo}_{2} \mathrm{Ru}_{4}(\mathrm{CO})_{12}(\mu-\mathrm{PPh})_{2}$ (8), which is isostructural with 4 but not with 3 , as indicated by $\mathrm{X}$-ray diffraction measurements. In connection with the tungsten-ruthenium and molybdenum-ruthenium phosphinidene clusters that we synthesized, Schauer and Carty have described the preparation of both a triruthenium phosphinidene cluster $\left[\mathrm{Ru}_{3}(\mathrm{CO})_{9}(\mu\right.$ $\left.\mathrm{H})\left(\mu_{3}-\mathrm{PPh}\right)\right]^{-}$by deprotonation of $\mathrm{Ru}_{3}(\mathrm{CO})_{10}(\mu$ $\mathrm{H})(\mu-\mathrm{PHPh})[7]$ and a novel nickel-ruthenium cluster $\mathrm{Cp}_{2} \mathrm{Ni}_{2} \mathrm{Ru}_{3}(\mathrm{CO})_{9}\left(\mu_{5}-\mathrm{PPh}\right)$ via condensation of unsaturated trinuclear cluster $\mathrm{Ru}_{3}(\mathrm{CO})_{9}(\mu-\mathrm{H})\left(\mu-\mathrm{PPh}_{2}\right)$ with $[\mathrm{CpNi}(\mathrm{CO})]_{2}[8]$, respectively.

\section{Experimental procedure}

\subsection{General information and materials}

Infrared spectra were recorded on a Perkin-Elmer 2000 FT-IR spectrometer. ${ }^{1} \mathrm{H}$ and ${ }^{13} \mathrm{C}$ NMR spectra were recorded on a Bruker AM-400 and a Varian Unity-400 instrument, whereas ${ }^{31} \mathrm{P}$ NMR spectra were recorded on a Bruker AMX-300 instrument. Chemical shifts are quoted with respect to internal standard tetramethylsilane $\left({ }^{1} \mathrm{H}\right.$ and ${ }^{13} \mathrm{C}$ NMR) and external standard $85 \% \mathrm{H}_{3} \mathrm{PO}_{4}\left({ }^{31} \mathrm{P}\right.$ NMR). Mass spectra were obtained on JEOL-HX110 instrument operating in fast atom bombardment (FAB) mode. The phosphido bridged triruthenium complex $\mathrm{Ru}_{3}(\mathrm{CO})_{10}(\mu-\mathrm{H})(\mu$ $\mathrm{PPh}_{2}$ ) was prepared from the reaction of $\mathrm{Ru}_{3}(\mathrm{CO})_{12}$ with diphenylphosphine in THF with sodium benzophenone ketyl as catalyst followed by thermolysis at 50 to $55^{\circ} \mathrm{C}$ in heptane solution [9]. Molybdenum hydride complex $\mathrm{Cp}^{\star} \mathrm{Mo}(\mathrm{CO})_{3} \mathrm{H}$ was prepared from reaction of (para-xylene) $\mathrm{Mo}(\mathrm{CO})_{3}$ with pentamethylcyclopentadiene [10]. All reactions were performed under a nitrogen atmosphere using deoxygenated solvents dried with an appropriate reagent. The progress of reactions was monitored by analytical thin-layer chromatography ( 5735 Kieselgel $60 \mathrm{~F}_{254}$, E. Merck) and the products were separated on thin-layer chromatographic plates (Kieselgel $60 \mathrm{~F}_{254}$, E. Merck). Elemental analyses were performed at the NSC Regional Instrumentation Center at National Cheng Kung University, Tainan, Taiwan.

\subsection{Reaction of $R u_{3}(C O)_{10}(\mu-H)\left(\mu-P P h_{2}\right)$ with $C^{\star} M o-$ $(\mathrm{CO})_{3} \mathrm{H}$}

In a $100 \mathrm{ml}$ round bottom reaction flask, the phosphido complex $\mathrm{Ru}_{3}(\mathrm{CO})_{10}(\mu-\mathrm{H})\left(\mu-\mathrm{PPh}_{2}\right)(100 \mathrm{mg}$, $0.128 \mathrm{mmol})$ and molybdenum hydride $\mathrm{Cp}^{\star} \mathrm{Mo}(\mathrm{CO})_{3} \mathrm{H}$ ( $160 \mathrm{mg}, 0.40 \mathrm{mmol}$ ) were dissolved in a toluene solution $(50 \mathrm{ml})$ and kept at reflux for $20 \mathrm{~min}$. The colour changed gradually from orange to dark brown. After the solution was cooled to room temperature, the solvent was evaporated in vacuo and the residue was separated by thin layer chromatography (dichloro- 
TABLE 1. Experimental data for the X-ray diffraction studies of complexes 5 and 8

\begin{tabular}{|c|c|c|}
\hline Compound & 5 & 8 \\
\hline Formula & $\mathrm{C}_{32} \mathrm{H}_{25} \mathrm{O}_{10} \mathrm{P}_{1} \mathrm{Mo}_{1} \mathrm{Ru}_{3}$ & $\mathrm{C}_{45} \mathrm{H}_{42} \mathrm{Cl}_{2} \mathrm{O}_{12} \mathrm{P}_{2} \mathrm{Mo}_{2} \mathrm{Ru}_{4}$ \\
\hline Mol. wt. & 999.66 & 1503.83 \\
\hline Crystal system & Monoclinic & Monoclinic \\
\hline Space group & $P 2_{1}$ & $C 2 / c$ \\
\hline$a(\AA)$ & $10.708(2)$ & $45.333(7)$ \\
\hline$b(\AA)$ & $10.848(4)$ & $10.185(2)$ \\
\hline$c(\AA)$ & $14.885(2)$ & $21.915(8)$ \\
\hline$\beta\left({ }^{\circ}\right)$ & $97.63(2)$ & $93.82(3)$ \\
\hline$U\left(\AA^{3}\right)$ & $1713.8(8)$ & $10096(4)$ \\
\hline Temperature & $25^{\circ} \mathrm{C}$ & $25^{\circ} \mathrm{C}$ \\
\hline$Z$ & 2 & 8 \\
\hline$D_{c}\left(\mathrm{~g} \mathrm{~cm}^{-3}\right)$ & 1.937 & 1.979 \\
\hline$F(000)$ & 972 & 5856 \\
\hline Crystal size, mm. & $0.20 \times 0.45 \times 0.45$ & $0.25 \times 0.50 \times 0.50$ \\
\hline$h, k, l$ ranges & $-1212,012,017$ & $-5151,011,024$ \\
\hline$\mu\left(\mathrm{mm}^{-1}\right)$ & 1.68 & 1.84 \\
\hline Absorption $\left(T_{\min } \& T_{\max }\right)$ & $0.80,1.00$ & $0.93,1.00$ \\
\hline No. of unique data $\left(2 \theta_{\max }\right)$ & $3180(50)$ & $7898(48)$ \\
\hline data with $I>2 \sigma(I)$ & 3025 & 6135 \\
\hline No. of atoms & 72 & 109 \\
\hline No. of variables & 432 & 577 \\
\hline$R ; R w$ & $0.021 ; 0.019$ & $0.035 ; 0.030$ \\
\hline G.O.F. & 2.09 & 2.22 \\
\hline
\end{tabular}

methane: hexane $=1: 7$ ), giving $13 \mathrm{mg}$ of red $\mathrm{Ru}_{4}(\mathrm{CO})_{13}\left(\mu_{3}-\mathrm{PPh}\right)(0.015 \mathrm{mmol}, 12 \%), 10 \mathrm{mg}$ of green $\mathrm{Ru}_{5}(\mathrm{CO})_{15}\left(\mu_{4}-\mathrm{PPh}\right)(0.01 \mathrm{mmol}, 7 \%), 47 \mathrm{mg}$ of orange $\mathrm{Cp}^{\star} \mathrm{MoRu}_{2}(\mathrm{CO})_{8}(\mu-\mathrm{H})\left(\mu_{3}-\mathrm{PPh}\right)(7,0.063 \mathrm{mmol}, 42 \%)$, $6 \mathrm{mg}$ of black phosphido cluster $\mathrm{Cp}^{\star} \mathrm{MoRu}_{3}(\mathrm{CO})_{10}(\mu$ $\mathrm{H})_{2}\left(\mu-\mathrm{PPh}_{2}\right)(5,0.006 \mathrm{mmol}, 4.7 \%)$ and $15 \mathrm{mg}$ of darkred cluster $\mathrm{Cp}^{\star} \mathrm{MoRu}_{3}(\mathrm{CO})_{10}\left(\mu_{3}-\mathrm{H}\right)\left(\mu_{3}-\mathrm{PPh}\right) \quad(6$, $0.016 \mathrm{mmol}, 13 \%)$ in the order of their elution.

Spectral data of 5: MS (FAB, $\left.{ }^{98} \mathrm{Mo},{ }^{102} \mathrm{Ru}\right), m / z$ $1006\left(\mathrm{M}^{+}\right)$; IR $\left(\mathrm{C}_{6} \mathrm{H}_{12}\right) \nu(\mathrm{CO}): 2072 \mathrm{~s}, 2021 \mathrm{vs}, 2006 \mathrm{vw}$, $1997 \mathrm{~m}, 1974 \mathrm{~s}, 1865 \mathrm{w}, \mathrm{br}, 1805 \mathrm{w}$, br $\mathrm{cm}^{-1} ;{ }^{1} \mathrm{H}$ NMR $\left(\mathrm{CD}_{2} \mathrm{Cl}_{2}, \mathrm{RT}\right): \delta$ 7.91-6.96 (m, $\left.10 \mathrm{H}\right), 1.98(\mathrm{~s}, 15 \mathrm{H})$, $-13.65\left(\mathrm{~d}, J_{\mathrm{P}-\mathrm{H}}=19.6 \mathrm{~Hz}\right),-19.52\left(\mathrm{~d}, J_{\mathrm{P}-\mathrm{H}}=\right.$ $11.2 \mathrm{~Hz}) .{ }^{13} \mathrm{C}$ NMR $\left(\mathrm{CD}_{2} \mathrm{Cl}_{2}, \mathrm{RT}\right): \mathrm{CO}, \delta 242.5$ (d, 2C, $\left.J_{\mathrm{P}-\mathrm{C}}=39 \mathrm{~Hz}\right), 237.2(\mathrm{~s}, 1 \mathrm{C}), 199.2(\mathrm{~s}, 2 \mathrm{C}), 195.5(\mathrm{~s}, 1 \mathrm{C})$, $192.9(\mathrm{~s}, 2 \mathrm{C}), 192.4$ (s, 2C). ${ }^{31} \mathrm{P}$ NMR $\left(\mathrm{CD}_{2} \mathrm{Cl}_{2}, \mathrm{RT}\right): \delta$ 367.1 (s). Elemental analysis: Found: $\mathrm{C}, 38.18 ; \mathrm{H}, 2.80$. $\mathrm{C}_{32} \mathrm{H}_{27} \mathrm{O}_{10} \mathrm{PMoRu}_{3}$ calcd.: $\mathrm{C}, 38.37 ; \mathrm{H}, 2.72 \%$.

Spectral data of 6: MS (FAB, $\left.{ }^{98} \mathrm{Mo},{ }^{102} \mathrm{Ru}\right), m / z$ $928\left(\mathrm{M}^{+}\right)$; IR $\left(\mathrm{C}_{6} \mathrm{H}_{12}\right) \nu(\mathrm{CO}): 2063 \mathrm{~s}, 2026 \mathrm{vs}, 2000 \mathrm{vw}$, $1991 \mathrm{~m}, 1973 \mathrm{~s}, 1962 \mathrm{~m}, 1893 \mathrm{w}, 1870 \mathrm{~m}, 1796 \mathrm{~m} \mathrm{~cm}^{-1} ;{ }^{1} \mathrm{H}$ NMR ( $\left.\mathrm{CDCl}_{3}, \mathrm{RT}\right): \delta 8.19-8.11(\mathrm{~m}, 2 \mathrm{H}), 7.66(\mathrm{~m}, 3 \mathrm{H})$, $1.80(\mathrm{~s}, 15 \mathrm{H}),-19.14\left(\mathrm{~d}, J_{\mathrm{P}-\mathrm{H}}=11.2 \mathrm{~Hz}\right) .{ }^{13} \mathrm{C}$ NMR $\left(\mathrm{CDCl}_{3}, \mathrm{RT}\right): \mathrm{CO}, \delta 261.7(\mathrm{~s}, 1 \mathrm{C}), 245.0\left(\mathrm{~d}, 1 \mathrm{C}, J_{\mathrm{P}-\mathrm{C}}=\right.$ $30 \mathrm{~Hz}), 222.5\left(\mathrm{~d}, 1 \mathrm{C}, J_{\mathrm{P}-\mathrm{C}}=14 \mathrm{~Hz}\right), 198.5(\mathrm{~s}, 1 \mathrm{C})$, $197.6\left(\mathrm{~d}, 3 \mathrm{C}, J_{\mathrm{P}_{31} \mathrm{C}}=8 \mathrm{~Hz}\right), 195.7(\mathrm{~s}, 1 \mathrm{C}), 192.2(\mathrm{~s}, 1 \mathrm{C})$, 191.9 (s, 1C). ${ }^{31} \mathrm{P}$ NMR (CDCl 3 , RT): $\delta 410.7(\mathrm{~s})$. Elemental analysis. Found: $\mathrm{C}, 32.25 ; \mathrm{H}, 2.32 . \mathrm{C}_{27} \mathrm{H}_{23^{-}}$ $\mathrm{Cl}_{2} \mathrm{O}_{10} \mathrm{PMoRu}_{3}$ calcd.: $\mathrm{C}, 32.16 ; \mathrm{H}, 2.30 \%$.
Spectral data of 7: MS (FAB, $\left.{ }^{98} \mathrm{Mo},{ }^{102} \mathrm{Ru}\right), m / z$ $770\left(\mathrm{M}^{+}\right)$; IR $\left(\mathrm{C}_{6} \mathrm{H}_{12}\right) \nu(\mathrm{CO})$ : 2082s, 2061vs, 2021vs, $1995 \mathrm{~m}, 1867 \mathrm{w}$, br, $1819 \mathrm{w}$, br cm ${ }^{-1} ;{ }^{1} \mathrm{H}$ NMR $\left(\mathrm{CDCl}_{3}\right.$, RT): $\delta 7.88(\mathrm{~m}, 2 \mathrm{H}), 7.64(\mathrm{~m}, 3 \mathrm{H}), 1.84(\mathrm{~s}, 15 \mathrm{H})$, $-19.04\left(\mathrm{~d}, 1 \mathrm{H}, J_{\mathrm{P}-\mathrm{H}}=14.3 \mathrm{~Hz}\right) .{ }^{13} \mathrm{C}$ NMR $\left(\mathrm{CD}_{2} \mathrm{Cl}_{2}\right.$, $213 \mathrm{~K})$ : CO, $\delta 239.9(\mathrm{~s}, 2 \mathrm{C}), 197.3\left(\mathrm{~d}, 2 \mathrm{C}, J_{\mathrm{P}-\mathrm{C}}=43 \mathrm{~Hz}\right)$, $192.3\left(\mathrm{~d}, 2 \mathrm{C}, J_{\mathrm{P}-\mathrm{C}}=7 \mathrm{~Hz}\right), 192.1\left(\mathrm{~d}, 2 \mathrm{C}, J_{\mathrm{P}-\mathrm{C}}=4 \mathrm{~Hz}\right)$; $\delta 135.9\left(\mathrm{~d}, 1 \mathrm{C}, J_{\mathrm{P}-\mathrm{C}}\right)=14 \mathrm{~Hz}, 135.2(\mathrm{~d}, 2 \mathrm{C}), J_{\mathrm{P}_{-} \mathrm{C}}=$ $11 \mathrm{~Hz}), 131.1\left(\mathrm{~d}, 1 \mathrm{C}, J_{\mathrm{P}-\mathrm{C}}=4 \mathrm{~Hz}\right), 129.4\left(\mathrm{~d}, 2 \mathrm{C}, J_{\mathrm{P}-\mathrm{C}}\right.$ $=11 \mathrm{~Hz}) .{ }^{31} \mathrm{P} \mathrm{NMR}\left(\mathrm{CDCl}_{3}, \mathrm{RT}\right): \delta 299.9(\mathrm{~s})$. Elemental analysis. Found: $\mathrm{C}, 37.60 ; \mathrm{H}, 2.89 . \mathrm{C}_{24} \mathrm{H}_{21} \mathrm{O}_{8} \mathrm{PMo}$ $\mathrm{Ru}_{2}$ calcd.: C, 37.61; H, 2.76\%.

\subsubsection{Thermolysis of 5}

A toluene solution $(30 \mathrm{ml})$ of $5(50 \mathrm{mg}, 0.050 \mathrm{mmol})$ was heated to reflux for $20 \mathrm{~min}$, during which the colour changed from dark-brown to red. The solvent was evaporated and the residue separated by thin layer chromatography (dichloromethane:hexane $=1: 1$ ) to afford $25 \mathrm{mg}$ of $6(0.027 \mathrm{mmol}, 54 \%)$ as red-orange powder.

\subsubsection{Reaction of 6 with carbon monoxide}

A toluene solution $(30 \mathrm{ml})$ of $6(60 \mathrm{mg}, 0.065 \mathrm{mmol})$ was refluxed under $1 \mathrm{~atm}$ of carbon monoxide for 15 min, during which the colour changed from red to orange. The solvent was evaporated and the residue was separated by thin layer chromatography (dichloromethane:hexane $=1: 7$ ), giving $3 \mathrm{mg}$ of $\mathrm{Ru}_{3}(\mathrm{CO})_{12}$ 
$(0.012 \mathrm{mmol})$ and $37 \mathrm{mg}$ of $7(0.049 \mathrm{mmol}, 75 \%)$ as orange powder.

\subsubsection{Thermolysis of 7}

A toluene solution $(30 \mathrm{ml})$ of $7(100 \mathrm{mg}, 0.099 \mathrm{mmol})$ was heated to reflux for two hours, during which the colour changed from orange-red to dark-brown. After allowing the solution to cool to room temperature, the solvent was evaporated and the residue was separated by thin layer chromatography (dichloromethane:hexane $=1: 1)$, giving $20 \mathrm{mg}$ of orange-red $7(0.026 \mathrm{mmol})$, $4 \mathrm{mg}$ of red $6(0.004 \mathrm{mmol}, 3 \%)$ and $45 \mathrm{mg}$ of darkbrown $\mathrm{Cp}^{\star} \mathrm{Mo}_{2} \mathrm{Ru}_{4}(\mathrm{CO})_{12}(\mu-\mathrm{PPh})_{2} \quad(8,0.032 \mathrm{mmol}$, $49 \%$ ). Crystals of 8 suitable for $X$-ray analysis were obtained by recrystallization from a layered solution of dichloromethane-methanol at room temperature.

Spectral data of 8: MS (FAB, $\left.{ }^{98} \mathrm{Mo},{ }^{102} \mathrm{Ru}\right), m / z$ $1426\left(\mathrm{M}^{+}\right)$; IR $\left(\mathrm{C}_{6} \mathrm{H}_{12}\right) \nu(\mathrm{CO}): 2051 \mathrm{vs}, 2019 \mathrm{~m}, 1996 \mathrm{~s}$, 1974w, 1960m, 1944w, 1933w, 1917w, 1863vw, br, 1791w, br $\mathrm{cm}^{-1} ;{ }^{1} \mathrm{H}$ NMR $\left(\mathrm{CDCl}_{3}, \mathrm{RT}\right): \delta 7.77-7.01(\mathrm{~m}, 10 \mathrm{H})$, $1.88(\mathrm{~s}, 15 \mathrm{H}), 1.65(\mathrm{~s}, 15 \mathrm{H}) .{ }^{13} \mathrm{C}$ NMR $\left(\mathrm{CDCl}_{3}, 218 \mathrm{~K}\right)$ : $\mathrm{CO}, \delta 271.5\left(\mathrm{~d}, J_{\mathrm{P}-\mathrm{H}}=8.5 \mathrm{~Hz}\right), 256.0\left(\mathrm{~d}, J_{\mathrm{P}-\mathrm{H}}=8 \mathrm{~Hz}\right)$, $235.2\left(\mathrm{~d}, J_{\mathrm{P}-\mathrm{H}}=8 \mathrm{~Hz}\right), 231.0,217.9,211.8,203.8(\mathrm{dd}$, $J_{\mathrm{P}-\mathrm{H}}=33$ and $\left.9.7 \mathrm{~Hz}\right), 200.5,200.3\left(\mathrm{~d}, J_{\mathrm{P}-\mathrm{H}}=29.6 \mathrm{~Hz}\right)$, 193.4, 190.6, 184.3. ${ }^{31}$ P NMR $\left(\mathrm{CDCl}_{3}, \mathrm{RT}\right): \delta 463.8$ (s), 382.5 (s). Elemental analysis. Found: $\mathrm{C}, 36.17$; H, 2.98. $\mathrm{C}_{45} \mathrm{H}_{42} \mathrm{Cl}_{2} \mathrm{O}_{12} \mathrm{P}_{2} \mathrm{Mo}_{2} \mathrm{Ru}_{4}$ calcd.: $\mathrm{C}, 35.94 ; \mathrm{H}, 2.82 \%$.

\subsection{X-ray crystallography}

Lattice parameters of 5 were determined from 25 randomly selected high angle reflections with $2 \theta$ angles in the range 19.00-25.00. The space group $P 2_{1}$ was identified on the basis of systematic absences and intensity statistics. All reflections were corrected for Lorentz, polarization and absorption effects. The absorption corrections were made by the $\Psi$ scan method and the minimum and maximum transmission factors were 0.796 and 1.000 , respectively. All data reduction and refinement were performed using the NRCCSDP-VAX packages. The structures were solved by direct method and refined by least squares cycle; all non-hydrogen atoms were refined with anisotropic thermal parameters. The position of the bridging hydride ligands was obtained from a difference Fourier synthesis and refined accordingly. The hydrogen atoms of the phenyl and pentamethylcyclopentadienyl groups were calculated in the idealized positions with a fixed temperature coefficient $\left(U_{\mathrm{H}}=U_{\mathrm{C}}+0.01 \AA^{2}\right)$ and included in the structure factor calculation.

Lattice parameters of 8 were determined from 25 randomly selected high angle reflections with $2 \theta$ angles in the range 18.64-25.26; the space group $C 2 / c$ was identified from the systematic absences and centric intensity distribution of the data, and confirmed by successfully solving the crystal structure. The absorption corrections were made by $\Psi$ scan method and the minimum and maximum transmission factors are 0.925 and 1.000 , respectively. All data reduction and refinement were performed using the NRCC-SDP-VAX packages. In the final stage of refinement, a $\mathrm{CH}_{2} \mathrm{Cl}_{2}$ molecule that came from the solvent mixture of recrys-

TABLE 2. Atomic coordinates and equivalent isotropic displacement coefficients for 5

\begin{tabular}{|c|c|c|c|c|}
\hline & $x$ & $y$ & $z$ & $B_{\text {iso }}$ \\
\hline Mo & $-0.28078(5)$ & $-0.54439(6)$ & $-0.20248(3)$ & $2.333(21)$ \\
\hline Ru1 & $-0.37158(5)$ & -0.78091 & $-0.13232(3)$ & $2.467(20)$ \\
\hline Ru2 & $-0.42350(5)$ & $-0.72791(5)$ & $-0.31728(3)$ & $2.329(20)$ \\
\hline Ru3 & $-0.54922(4)$ & $-0.58321(5)$ & $-0.19797(3)$ & $2.213(20)$ \\
\hline $\mathbf{P}$ & $-0.64113(15)$ & $-0.72150(18)$ & $-0.30693(10)$ & $2.63(7)$ \\
\hline O1 & $-0.3382(5)$ & $-0.7601(5)$ & $0.0741(3)$ & $6.3(3)$ \\
\hline $\mathrm{O} 2$ & $-0.1441(4)$ & $-0.9475(5)$ & $-0.1312(4)$ & $6.3(3)$ \\
\hline $\mathrm{O} 3$ & $-0.5219(4)$ & $-1.0181(4)$ & $-0.1370(3)$ & $3.93(23)$ \\
\hline $\mathrm{O} 4$ & $-0.4066(5)$ & $-1.0045(4)$ & $-0.3411(3)$ & $5.7(3)$ \\
\hline 05 & $-0.4182(5)$ & $-0.6621(6)$ & $-0.5143(3)$ & $6.1(3)$ \\
\hline 06 & $-0.6716(5)$ & $-0.3510(5)$ & $-0.2806(3)$ & $5.8(3)$ \\
\hline 07 & $-0.7079(4)$ & $-0.5632(5)$ & $-0.0468(3)$ & $4.58(25)$ \\
\hline 08 & $-0.1367(4)$ & $-0.7621(5)$ & $-0.2982(3)$ & $4.29(25)$ \\
\hline O9 & $-0.3921(4)$ & $-0.4377(5)$ & $-0.0353(3)$ & $4.6(3)$ \\
\hline O10 & $-0.0944(5)$ & $-0.6513(5)$ & $-0.0459(4)$ & $6.9(3)$ \\
\hline $\mathrm{C} 1$ & $-0.3514(6)$ & $-0.7645(6)$ & $-0.0036(4)$ & $3.5(3)$ \\
\hline $\mathrm{C} 2$ & $-0.2242(6)$ & $-0.8814(7)$ & $-0.1327(4)$ & $3.9(3)$ \\
\hline C3 & $-0.4710(6)$ & $-0.9281(6)$ & $-0.1376(4)$ & $2.9(3)$ \\
\hline $\mathrm{C} 4$ & $-0.4127(6)$ & $-0.9014(7)$ & $-0.3327(4)$ & $3.7(3)$ \\
\hline C5 & $-0.4261(6)$ & $-0.6841(6)$ & $-0.4396(4)$ & $3.4(3)$ \\
\hline C6 & $-0.6240(6)$ & $-0.4391(7)$ & $-0.2506(4)$ & $3.4(3)$ \\
\hline C7 & $-0.6490(5)$ & $-0.5705(6)$ & $-0.1055(4)$ & $2.9(3)$ \\
\hline $\mathrm{C} 8$ & $-0.2336(6)$ & $-0.7191(7)$ & $-0.2871(4)$ & $3.7(3)$ \\
\hline $\mathrm{C} 9$ & $-0.3823(6)$ & $-0.4906(6)$ & $-0.1022(4)$ & $3.6(3)$ \\
\hline $\mathrm{C} 10$ & $-0.1721(6)$ & $-0.6247(7)$ & $-0.1043(4)$ & $4.1(3)$ \\
\hline $\mathrm{C} 11$ & $-0.7211(5)$ & $-0.8600(6)$ & $-0.2759(4)$ & $2.9(3)$ \\
\hline $\mathrm{C} 12$ & $-0.7856(6)$ & $-0.8636(7)$ & $-0.2008(5)$ & $4.0(4)$ \\
\hline $\mathrm{C} 13$ & $-0.8436(7)$ & $-0.9702(9)$ & $-0.1799(5)$ & $5.9(5)$ \\
\hline $\mathrm{Cl} 4$ & $-0.8391(7)$ & $-1.0737(8)$ & $-0.2309(5)$ & $5.6(4)$ \\
\hline $\mathrm{C} 15$ & $-0.7783(6)$ & $-1.0705(7)$ & $-0.3062(5)$ & $4.8(4)$ \\
\hline C16 & $-0.7205(6)$ & $-0.9650(7)$ & $-0.3288(4)$ & $3.7(3)$ \\
\hline C17 & $-0.7492(6)$ & $-0.6634(6)$ & $-0.4038(4)$ & $2.9(3)$ \\
\hline $\mathrm{C} 18$ & $-0.8615(6)$ & $-0.7208(7)$ & $-0.4370(4)$ & $3.9(3)$ \\
\hline C19 & $-0.9358(7)$ & $-0.6731(8)$ & $-0.5128(5)$ & $5.3(4)$ \\
\hline $\mathrm{C} 20$ & $-0.9036(7)$ & $-0.5685(9)$ & $-0.5536(4)$ & $5.7(4)$ \\
\hline $\mathrm{C} 21$ & $-0.7967(7)$ & $-0.5089(9)$ & $-0.5199(4)$ & $5.6(5)$ \\
\hline $\mathrm{C} 22$ & $-0.7186(6)$ & $-0.5580(7)$ & $-0.4469(4)$ & $4.1(4)$ \\
\hline $\mathrm{C} 23$ & $-0.1684(6)$ & $-0.4514(6)$ & $-0.3112(4)$ & $3.3(3)$ \\
\hline $\mathrm{C} 24$ & $-0.2735(6)$ & $-0.3770(6)$ & $-0.3026(4)$ & $3.3(3)$ \\
\hline $\mathrm{C} 25$ & $-0.2615(6)$ & $-0.3310(6)$ & $-0.2125(4)$ & $3.2(3)$ \\
\hline $\mathrm{C} 26$ & $-0.1475(7)$ & $-0.3817(7)$ & $-0.1650(5)$ & $4.4(4)$ \\
\hline $\mathrm{C} 27$ & $-0.0914(6)$ & $-0.4560(7)$ & $-0.2276(5)$ & $3.9(3)$ \\
\hline $\mathrm{C} 28$ & $-0.1381(7)$ & $-0.5035(8)$ & $-0.3989(5)$ & $5.6(5)$ \\
\hline C29 & $-0.3703(7)$ & $-0.3359(7)$ & $-0.3781(5)$ & $4.5(4)$ \\
\hline $\mathrm{C} 30$ & $-0.3397(8)$ & $-0.2307(7)$ & $-0.1790(5)$ & $5.5(4)$ \\
\hline C31 & $-0.0921(9)$ & $-0.3426(8)$ & $-0.0714(5)$ & $7.3(5)$ \\
\hline $\mathrm{C} 32$ & $0.0382(6)$ & $-0.5120(9)$ & $-0.2083(6)$ & $7.4(5)$ \\
\hline H1 & $-0.453(4)$ & $-0.574(5)$ & $-0.293(3)$ & $4.1(13)$ \\
\hline $\mathrm{H} 2$ & $-0.506(4)$ & $-0.725(5)$ & $-0.127(3)$ & 4.1(11) \\
\hline
\end{tabular}


tallization was located on the difference Fourier map; the carbon and chlorine atoms were then included in the analysis and refined accordingly. The combined

TABLE 3. Atomic coordinates and equivalent isotropic displacement coefficients for 8

\begin{tabular}{|c|c|c|c|c|}
\hline & $x$ & $y$ & $z$ & $B_{\text {iso }}$ \\
\hline Ru1 & $0.127167(12)$ & $0.04337(6)$ & $0.182141(24)$ & $2.244(23)$ \\
\hline Ru2 & $0.104508(12)$ & $-0.19708(6)$ & $0.215971(24)$ & $2.436(25)$ \\
\hline Ru3 & $0.133568(12)$ & $-0.05405(6)$ & $0.313322(23)$ & $2.69(3)$ \\
\hline Ru4 & $0.182483(12)$ & $-0.01673(6)$ & $0.246046(23)$ & $2.232(24)$ \\
\hline Mo1 & $0.157945(12)$ & $-0.19053(6)$ & $0.143955(24)$ & $2.085(25)$ \\
\hline Mo2 & $0.066946(13)$ & $-0.03662(8)$ & $0.13343(3)$ & $3.29(3)$ \\
\hline P1 & $0.15510(4)$ & $-0.22254(19)$ & $0.25071(8)$ & $2.36(8)$ \\
\hline $\mathrm{P} 2$ & $0.17303(4)$ & $0.02659(18)$ & $0.14520(7)$ & $2.33(8)$ \\
\hline 01 & $0.12348(12)$ & $0.2881(5)$ & $0.25969(23)$ & $5.1(3)$ \\
\hline $\mathrm{O} 2$ & $0.11142(12)$ & $0.2384(6)$ & $0.0835(3)$ & $6.5(3)$ \\
\hline $\mathrm{O} 3$ & $0.09684(9)$ & $-0.1411(5)$ & $0.07886(18)$ & $2.91(22)$ \\
\hline O4 & $0.08933(12)$ & $-0.4815(5)$ & $0.19665(25)$ & $5.8(3)$ \\
\hline O5 & $0.06688(10)$ & $-0.1881(6)$ & $0.32475(21)$ & $5.8(3)$ \\
\hline O6 & $0.03618(11)$ & $-0.2595(7)$ & $0.1986(3)$ & $7.4(4)$ \\
\hline O7 & $0.09399(12)$ & $0.1442(6)$ & $0.37120(23)$ & $6.3(3)$ \\
\hline O8 & $0.12962(12)$ & $-0.2180(6)$ & $0.42632(21)$ & $5.8(3)$ \\
\hline O9 & $0.18682(12)$ & $0.0552(7)$ & $0.38527(22)$ & 7.1(4) \\
\hline $\mathrm{O} 10$ & $0.24514(10)$ & $-0.1095(6)$ & $0.26429(25)$ & $5.5(3)$ \\
\hline O11 & $0.20111(12)$ & $0.2665(5)$ & $0.26789(24)$ & $5.4(3)$ \\
\hline $\mathrm{O} 12$ & $0.05721(11)$ & $0.1282(6)$ & $0.25009(24)$ & $6.2(3)$ \\
\hline $\mathrm{Cl}$ & $0.12491(15)$ & $0.1912(7)$ & $0.2332(3)$ & $3.5(3)$ \\
\hline $\mathrm{C} 2$ & $0.11462(15)$ & $0.1538(7)$ & $0.1173(3)$ & $3.7(4)$ \\
\hline C3 & $0.11716(14)$ & $-0.1485(7)$ & $0.1213(3)$ & $2.5(3)$ \\
\hline $\mathrm{C} 4$ & $0.09538(15)$ & $-0.3758(8)$ & $0.2027(3)$ & $3.8(4)$ \\
\hline C5 & $0.08401(14)$ & $-0.1804(8)$ & $0.2889(3)$ & $3.6(4)$ \\
\hline C6 & $0.05367(15)$ & $-0.1889(8)$ & $0.1797(3)$ & $4.5(4)$ \\
\hline $\mathrm{C7}$ & $0.10853(15)$ & $0.0702(8)$ & $0.3477(3)$ & $3.7(4)$ \\
\hline $\mathrm{C} 8$ & $0.13113(15)$ & $-0.1550(8)$ & $0.3835(3)$ & $3.7(4)$ \\
\hline $\mathrm{C} 9$ & $0.17125(16)$ & $0.0190(8)$ & $0.3435(3)$ & $4.5(4)$ \\
\hline C10 & $0.22085(14)$ & $-0.0760(7)$ & $0.2578(3)$ & $3.2(3)$ \\
\hline C11 & $0.19452(15)$ & $0.1607(7)$ & $0.2598(3)$ & $3.3(3)$ \\
\hline C12 & $0.06314(15)$ & $0.0661(8)$ & $0.2093(3)$ & $3.9(4)$ \\
\hline $\mathrm{C} 13$ & $0.16831(14)$ & $-0.3574(7)$ & $0.3006(3)$ & $2.7(3)$ \\
\hline C14 & $0.14862(15)$ & $-0.4440(8)$ & $0.3234(3)$ & $3.9(4)$ \\
\hline C15 & $0.15809(17)$ & $-0.5407(8)$ & $0.3644(3)$ & $5.1(4)$ \\
\hline $\mathrm{C} 16$ & $0.18709(17)$ & $-0.5515(8)$ & $0.3822(3)$ & $4.3(4)$ \\
\hline $\mathrm{C} 17$ & $0.20665(16)$ & $-0.4683(8)$ & $0.3608(3)$ & $4.5(4)$ \\
\hline C18 & $0.19777(15)$ & $-0.3724(7)$ & $0.3184(3)$ & $3.9(4)$ \\
\hline C19 & $0.19223(14)$ & $0.1428(7)$ & $0.0993(3)$ & $2.6(3)$ \\
\hline $\mathrm{C} 20$ & $0.21908(14)$ & $0.1958(7)$ & $0.1197(3)$ & $3.1(3)$ \\
\hline $\mathrm{C} 21$ & $0.23286(15)$ & $0.2875(8)$ & $0.0855(3)$ & $4.3(4)$ \\
\hline $\mathrm{C} 22$ & $0.21980(18)$ & $0.3294(8)$ & $0.0318(3)$ & $4.9(4)$ \\
\hline $\mathrm{C} 23$ & $0.19288(18)$ & $0.2788(9)$ & $0.0106(3)$ & $5.1(4)$ \\
\hline $\mathrm{C} 24$ & $0.17899(15)$ & $0.1850(7)$ & $0.0440(3)$ & $3.7(4)$ \\
\hline $\mathrm{C} 25$ & $0.14994(15)$ & $-0.3623(7)$ & $0.0743(3)$ & $3.0(3)$ \\
\hline $\mathrm{C} 26$ & $0.17150(16)$ & $-0.4060(7)$ & $0.1204(3)$ & $3.4(3)$ \\
\hline $\mathrm{C} 27$ & $0.19682(14)$ & $-0.3273(7)$ & $0.1171(3)$ & $3.2(3)$ \\
\hline $\mathrm{C} 28$ & $0.19141(14)$ & $-0.2351(7)$ & $0.0690(3)$ & $3.2(3)$ \\
\hline $\mathrm{C} 29$ & $0.16224(15)$ & $-0.2563(7)$ & $0.0432(3)$ & $3.1(3)$ \\
\hline C30 & $0.12191(17)$ & $-0.4309(8)$ & $0.0555(3)$ & $5.0(4)$ \\
\hline C31 & $0.16805(20)$ & $-0.5245(8)$ & $0.1573(3)$ & $5.5(5)$ \\
\hline $\mathrm{C} 32$ & $0.22558(17)$ & $-0.3489(9)$ & $0.1514(3)$ & $5.3(5)$ \\
\hline $\mathrm{C} 33$ & $0.21453(17)$ & $-0.1486(8)$ & $0.0439(4)$ & $5.3(5)$ \\
\hline C34 & $0.14785(17)$ & $-0.1867(8)$ & $-0.0113(3)$ & $4.4(4)$ \\
\hline
\end{tabular}

TABLE 3 (continued)

\begin{tabular}{llllc}
\hline & $x$ & $y$ & \multicolumn{1}{l}{$z$} & \multicolumn{1}{l}{$B_{\text {iso }}$} \\
\hline C35 & $0.03824(17)$ & $0.1323(9)$ & $0.0883(4)$ & $5.9(5)$ \\
C36 & $0.01905(16)$ & $0.0330(11)$ & $0.1176(3)$ & $6.6(5)$ \\
C37 & $0.02073(17)$ & $-0.0819(12)$ & $0.0838(4)$ & $8.5(6)$ \\
C38 & $0.03835(17)$ & $-0.0688(10)$ & $0.0402(3)$ & $6.5(5)$ \\
C39 & $0.05014(15)$ & $0.0565(9)$ & $0.0398(3)$ & $4.4(4)$ \\
C40 & $0.0391(3)$ & $0.2684(12)$ & $0.0985(6)$ & $16.1(10)$ \\
C41 & $-0.00280(21)$ & $0.0512(19)$ & $0.1618(4)$ & $17.4(13)$ \\
C42 & $0.00060(24)$ & $-0.2037(14)$ & $0.0909(5)$ & $12.2(9)$ \\
C43 & $0.04464(24)$ & $-0.1782(13)$ & $-0.0058(5)$ & $11.0(8)$ \\
C44 & $0.06894(20)$ & $0.0974(13)$ & $-0.0093(4)$ & $9.7(8)$ \\
C45 & $0.4397(3)$ & $-0.0640(15)$ & $0.1548(8)$ & $21.4(14)$ \\
C11 & $0.47519(18)$ & $-0.0308(9)$ & $0.1778(4)$ & $40.5(11)$ \\
C12 & $0.4399(3)$ & $0.0021(8)$ & $0.0894(4)$ & $56.4(15)$ \\
\hline
\end{tabular}

TABLE 4. Selected bond distances $(\AA)$ and bond angles $\left(^{\circ}\right)$ for 5 (esd's in parentheses)

\begin{tabular}{|c|c|c|c|}
\hline Mo-Ru(1) & $2.981(1)$ & $\mathrm{Mo}-\mathrm{Ru}(2)$ & $2.920(1)$ \\
\hline Mo-Ru(3) & $2.915(1)$ & $\operatorname{Ru}(1)-\operatorname{Ru}(2)$ & $2.795(1)$ \\
\hline $\mathbf{R u}(1)-\mathbf{R u}(3)$ & $2.947(1)$ & $\operatorname{Ru}(2)-\operatorname{Ru}(3)$ & $2.840(1)$ \\
\hline $\mathbf{R u}(2)-\mathbf{P}$ & $2.357(2)$ & $\mathrm{Ru}(3)-\mathrm{P}$ & $2.329(2)$ \\
\hline Mo-C(8) & $2.367(7)$ & $\mathrm{Ru}(2)-\mathrm{C}(8)$ & $2.026(7)$ \\
\hline Mo-C(9) & $2.045(6)$ & $\mathrm{Ru}(3)-\mathrm{C}(9)$ & $2.358(7)$ \\
\hline Mo-C(10) & $1.949(6)$ & $\mathrm{Ru}(1)-\mathrm{C}(1)$ & $1.908(6)$ \\
\hline $\mathrm{Ru}(1)-\mathrm{C}(2)$ & $1.919(7)$ & $\mathrm{Ru}(1)-\mathrm{C}(3)$ & $1.915(7)$ \\
\hline $\mathrm{Ru}(2)-\mathrm{C}(4)$ & $1.901(7)$ & $\mathrm{Ru}(2)-C(5)$ & $1.878(6)$ \\
\hline $\mathrm{Ru}(3)-\mathrm{C}(6)$ & $1.880(7)$ & $\mathrm{Ru}(3)-\mathrm{C}(7)$ & $1.857(5)$ \\
\hline Mo-H(1) & $2.15(5)$ & $\mathrm{Ru}(2)-\mathrm{H}(1)$ & $1.75(6)$ \\
\hline $\mathrm{Ru}(3)-\mathrm{H}(1)$ & $1.86(4)$ & $\mathrm{Ru}(1)-\mathrm{H}(2)$ & $1.58(4)$ \\
\hline $\mathbf{R u}(3)-\mathbf{H}(2)$ & $1.89(5)$ & & \\
\hline Mo-Ru(1)-C(1) & $105.8(2)$ & Mo-Ru(1)-C(2) & $100.5(2)$ \\
\hline $\mathrm{Ru}(1)-\mathrm{Mo}-\mathrm{C}(10)$ & $62.8(2)$ & Mo-Ru(2)-C(5) & $109.5(2)$ \\
\hline$M o-R u(3)-C(6)$ & $103.7(2)$ & $\mathrm{Mo}-\mathrm{C}(8) \mathrm{O}(8)$ & $130.6(5)$ \\
\hline $\mathrm{Ru}(2)-\mathrm{C}(8) \mathrm{O}(8)$ & $146.4(6)$ & Mo-C(9)O(9) & $151.7(5)$ \\
\hline $\operatorname{Ru}(3)-C(9) O(9)$ & $125.4(5)$ & Mo-C(10)O(10) & $166.7(6)$ \\
\hline Mean $\mathbf{R u}-\mathrm{CO}$ (terminal) & $176.6(6)$ & & \\
\hline
\end{tabular}

TABLE 5. Selected bond distances $(\AA)$ and bond angles $\left(^{\circ}\right)$ for 8 (esd's in parentheses)

\begin{tabular}{lclr}
\hline $\mathrm{Ru}(1)-\mathrm{Ru}(2)$ & $2.776(1)$ & $\mathrm{Ru}(1)-\mathrm{Ru}(3)$ & $3.037(1)$ \\
$\mathrm{Ru}(1)-\mathrm{Ru}(4)$ & $2.856(1)$ & $\mathrm{Ru}(1)-\mathrm{Mo}(1)$ & $2.912(1)$ \\
$\mathrm{Ru}(1)-\mathrm{Mo}(2)$ & $2.978(1)$ & $\mathrm{Ru}(2)-\mathrm{Ru}(3)$ & $2.835(1)$ \\
$\mathrm{Ru}(2)-\mathrm{Mo}(1)$ & $2.979(1)$ & $\mathrm{Ru}(2)-\mathrm{Mo}(2)$ & $2.904(1)$ \\
$\mathrm{Ru}(3)-\mathrm{Ru}(4)$ & $2.770(1)$ & $\mathrm{Ru}(4)-\mathrm{Mo}(1)$ & $3.008(1)$ \\
$\mathrm{Ru}(1)-\mathrm{P}(2)$ & $2.287(2)$ & $\mathrm{Ru}(2)-\mathrm{P}(1)$ & $2.382(2)$ \\
$\mathrm{Ru}(3)-\mathrm{P}(1)$ & $2.441(2)$ & $\mathrm{Ru}(4)-\mathrm{P}(1)$ & $2.442(2)$ \\
$\mathrm{Ru}(4)-\mathrm{P}(2)$ & $2.266(2)$ & $\mathrm{Mo}(1)-\mathrm{P}(1)$ & $2.374(2)$ \\
$\mathrm{Mo}(1)-\mathrm{P}(2)$ & $2.314(2)$ & $\mathrm{Mo}(1)-\mathrm{C}(3)$ & $1.930(6)$ \\
$\mathrm{Ru}(1)-\mathrm{C}(3)$ & $2.393(6)$ & $\mathrm{Ru}(2)-\mathrm{C}(3)$ & $2.244(6)$ \\
$\mathrm{Mo}(2)-\mathrm{C}(3)$ & $2.575(6)$ & $\mathrm{Mo}(2)-\mathrm{O}(3)$ & $2.148(4)$ \\
$\mathrm{C}(3)-\mathrm{O}(3)$ & $1.267(7)$ & $\mathrm{Mo}(2)-\mathrm{C}(6)$ & $1.969(8)$ \\
$\mathrm{Ru}(2)-\mathrm{C}(6)$ & $2.389(7)$ & $\mathrm{Ru}(3)-\mathrm{C}(9)$ & $1.938(7)$ \\
$\mathrm{Ru}(4)-\mathrm{C}(9)$ & $2.257(7)$ & $\mathrm{Mo}(2)-\mathrm{C}(12)$ & $1.982(7)$ \\
$\mathrm{Mo}(1)-\mathrm{C}(3)-\mathrm{O}(3)$ & $147.1(5)$ & $\mathrm{Mo}(2)-O(3)-\mathrm{C}(3)$ & $94.3(4)$ \\
$\mathrm{Ru}(1)-\mathrm{C}(3)-\mathrm{Ru}(2)$ & $73.5(2)$ & $\mathrm{Mo}(2)-\mathrm{C}(6)-O(6)$ & $155.1(6)$ \\
$\mathrm{Ru}(3)-\mathrm{C}(9)-\mathrm{O}(9)$ & $148.7(6)$ & $\mathrm{Ru}(4)-\mathrm{C}(9)-O(9)$ & $128.7(5)$ \\
$\mathrm{Mo}(2)-\mathrm{C}(12)-\mathrm{O}(12)$ & $171.0(6)$ & $\mathrm{Ru}(2)-\mathrm{C}(5)-\mathrm{O}(5)$ & $163.7(6)$ \\
\hline
\end{tabular}


data collection and refinement parameters are given in Table 1. Atomic positional parameters for 5 and 8 are presented in Tables 2 and 3, whereas data of selected bond angles and lengths are given in Tables 4 and 5 , respectively. Tables of bond distances and angles, positional parameters of hydrogen atoms and anisotropic thermal parameters of nonhydrogen atoms and listings of the observed and calculated structural factors are available from YC.

\section{Results and discussion}

Treatment of triruthenium phosphido complex $\mathrm{Ru}_{3}(\mathrm{CO})_{10}(\mu-\mathrm{H})\left(\mu-\mathrm{PPh}_{2}\right)$ with excess $\mathrm{Cp}^{\star} \mathrm{Mo}(\mathrm{CO})_{3} \mathrm{H}$ in refluxing toluene $(20 \mathrm{~min})$ produced the tetranuclear, black phosphido cluster $\mathrm{Cp}^{\star} \mathrm{MoRu}_{3}(\mathrm{CO})_{10}(\mu$ $\mathrm{H})_{2}\left(\mu-\mathrm{PPh}_{2}\right)$ in small amount $(5,4.7 \%)$, phosphinidene complex Cp* $\mathrm{MoRu}_{3}(\mathrm{CO})_{10}\left(\mu_{3}-\mathrm{H}\right)\left(\mu_{3}-\mathrm{PPh}\right) \quad(6,13 \%)$ and trinuclear, orange phosphinidene cluster $\mathrm{Cp}^{\star} \mathrm{MoRu}_{2}(\mathrm{CO})_{8}(\mu-\mathrm{H})\left(\mu_{3}-\mathrm{PPh}\right)(7,43 \%)$, in addition to two homometallic phosphinidene complexes $\mathrm{Ru}_{4^{-}}$
$(\mathrm{CO})_{13}\left(\mu_{3}-\mathrm{PPh}\right)$ and $\mathrm{Ru}_{5}(\mathrm{CO})_{15}\left(\mu_{4}-\mathrm{PPh}\right)$. The latter homometallic phosphinidenes are evidently produced by self-condensation of the precursor $\mathrm{Ru}_{3}(\mathrm{CO})_{10}(\mu$ $\mathrm{H})\left(\mu-\mathrm{PPh}_{2}\right)$; the tetraruthenium complex $\mathrm{Ru}_{4}(\mathrm{CO})_{13^{-}}$ $\left(\mu_{3}-\mathrm{PPh}\right)$ was first prepared by Carty and coworkers via the thermal pyrolysis of $\mathrm{Ru}_{3}(\mathrm{CO})_{9}(\mu-\mathrm{H})\left(\mu-\mathrm{PPh}_{2}\right)$ in heptane solution [11], whereas the pentanuclear complex $\mathrm{Ru}_{5}(\mathrm{CO})_{15}\left(\mu_{4}-\mathrm{PPh}\right)$ was obtained from the reactions of $\mathrm{Ru}_{3}(\mathrm{CO})_{12}$ with $\mathrm{CpMn}(\mathrm{CO})_{2}\left(\mathrm{PCl}_{2} \mathrm{Ph}\right)$ in small quantity [12] and the reaction with an equimolar amount of $\mathrm{PPhH}_{2}$ in toluene [13].

\subsection{Characterization of 5}

This complex shows a molecular ion in its FAB mass spectrum and fragmentation ions corresponding to successive loss of $\mathrm{CO}$ ligands. The solution IR spectrum exhibits seven terminal $\mathrm{CO}$ stretching absorptions and two additional weak absorptions at 1865 and $1805 \mathrm{~cm}^{-1}$, indicating the presence of bridging $\mathrm{CO}$ ligands. The ${ }^{1} \mathrm{H}$ NMR spectrum shows two hydride signals at $\delta-13.65\left(\mathrm{~d}, J_{\mathrm{P}-\mathrm{H}}=19.6 \mathrm{~Hz}\right)$ and -19.52

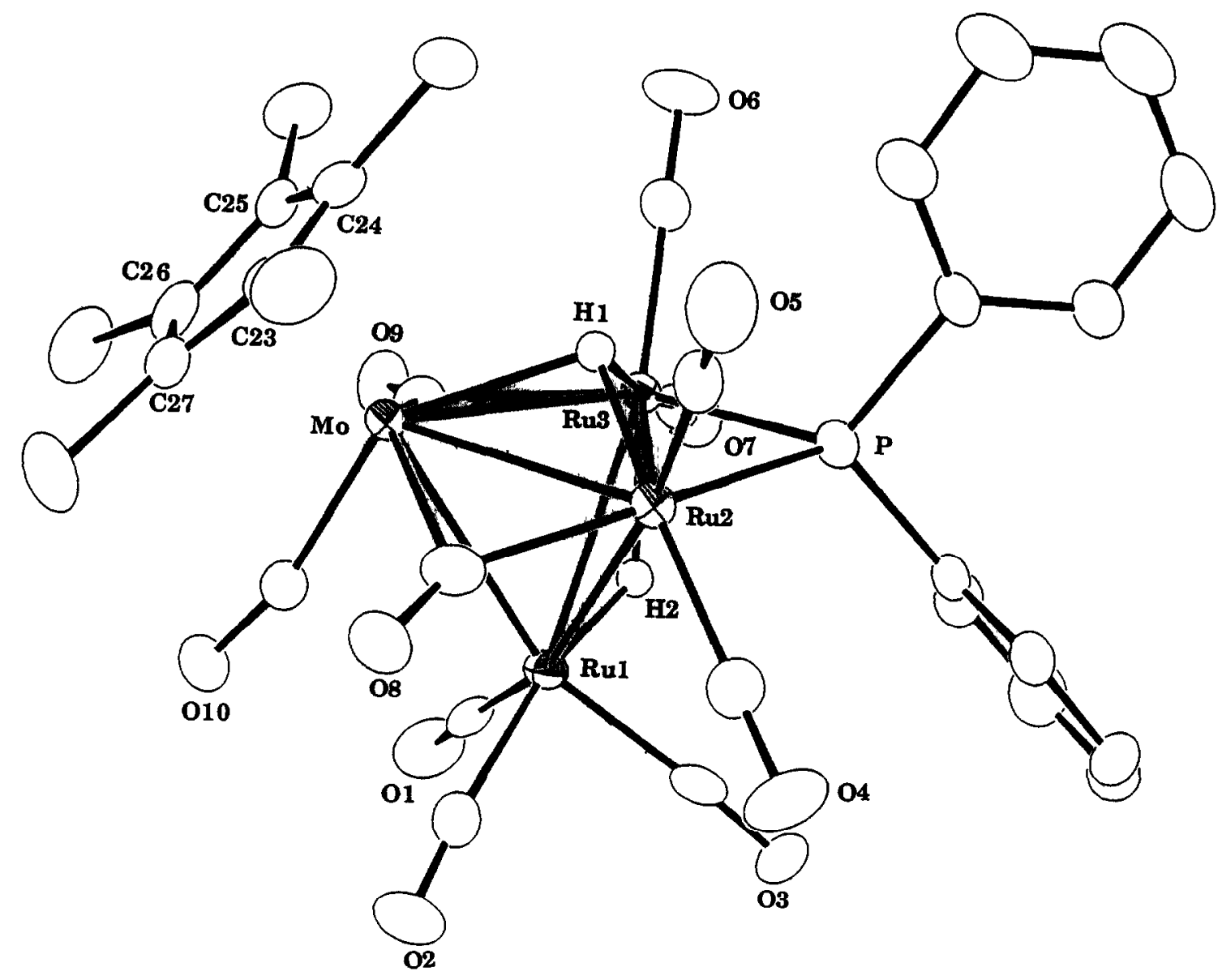

Fig. 1. Molecular structure of 5 and the atomic numbering scheme. 
(d, $J_{\mathrm{P}-\mathrm{H}}=11.2 \mathrm{~Hz}$ ) and several multiplets in the range $\delta$ 7.91-6.96, indicating the presence of two phenyl groups. The chemical shift value of signal at $\delta-19.52$ is normal for an edge-bridging hydride and the downfield chemical shift for the hydride at $\delta-13.65$ implies that it belongs to a rare, triply bridging hydride [14]. As these spectroscopic data do not uniquely define its molecular structure, a single-crystal $\mathrm{X}$-ray analysis was carried out.

The molecular diagram is shown in Fig. 1, and selected bond parameters are presented in Table 3 . The metal framework may be described as a distorted $\mathrm{MoRu}_{3}$ tetrahedron, with metal-metal bond distances being in the range 2.795(1)-2.981(1) $\AA$. The $R u(2)-$ $\mathrm{Ru}(3)$ edge $(2.840(1) \AA$ ), which is bridged by the phosphido ligand, is intermediate between the other two $\mathrm{Ru}-\mathrm{Ru}$ bonds, $\mathrm{Ru}(1)-\mathrm{Ru}(3)=2.947(1)$ and $\mathrm{Ru}(1)$ $\mathrm{Ru}(2)=2.795(1) \AA$. The $R u(2)-P$ and $R u(3)-P$ distances of $2.357(2)$ and $2.329(2) \AA$, respectively, are in the range observed for previously reported ruthenium clusters containing phosphido ligands [15]. For example, in $\mathrm{Ru}_{4}\left(\mu_{4}-\mathrm{C}_{2}\right)\left(\mu-\mathrm{PPh}_{2}\right)_{2}(\mathrm{CO})_{12}$, the corresponding value are $2.344(2)$ and $2.357(2) \AA$, respectively [15(b)]. In addition, there are eight terminal and two bridging $\mathrm{CO}$ ligands in the molecule. The bridging $\mathrm{CO}$ ligands lie on an extension of the metal triangle defined by the atoms $\mathrm{Mo}, \mathrm{Ru}(2)$ and $\mathrm{Ru}(3)$. The three $\mathrm{Ru}$ atoms are each coordinated by three, two and two terminal CO ligands, respectively; the $\mathrm{Mo}$ atom is bonded to a $\mathrm{Cp}^{\star}$ ligand and carries only one terminal CO ligand.

The most striking aspect of the structure of 5 is the position of hydride ligands. Both hydride ligands have been unambiguously located in a difference Fourier synthesis; one occupies the $\mathrm{Ru}(1)-\mathrm{Ru}(3)$ edge with $\mathrm{Ru}(1)-\mathrm{H}(2)=1.58(4)$ and $\mathrm{Ru}(3)-\mathrm{H}(2)=1.89(5) \AA$, and the second caps the $\mathrm{MoRu}_{2}$ triangle, which is surrounded by two edge bridging $\mathrm{CO}$ and phosphido ligands, with the respective bond distances being $\mathrm{Mo}$ $\mathrm{H}(1)=2.15(5), \mathrm{Ru}(2)-\mathrm{H}(1)=1.75(6)$ and $\mathrm{Ru}(3)-\mathrm{H}(1)$ $=1.86(4) \AA$. The elongation of the Mo-H(1) bond may be ascribed to unfavourable steric interaction of the
$\mathrm{Cp}^{\star}$ ligand with the Mo atom. The influence exerted on the $\mathrm{Ru}(1)-\mathrm{Ru}(3)$ bond by the edge-bridging hydride $\mathrm{H}(2)$ is noticeable; it is considerably elongated (2.947(1) $\AA$ ) relative to the $R u(1)-R u(2)$ bond (2.795(1) $\AA$ ) that possesses no bridging hydride. The coordination behaviour of the face-bridging hydride is comparable to that of the tetranuclear cluster complexes $\mathrm{Fe}_{3} \mathrm{Pt}(\mathrm{CO})_{10}\left(\mu_{3}-\mathrm{H}\right)\left(\mu_{3}-\mathrm{COMe}\right)\left(\mathrm{PPh}_{3}\right)$ [16] and $\mathrm{Co}_{3} \mathrm{Fe}(\mathrm{CO})_{9}\left(\mu_{3}-\mathrm{H}\right)\left[\mathrm{P}(\mathrm{OMe})_{3}\right]_{3}$ [17]. A few face-bridging hydrides have also been observed for trinuclear cluster and higher nuclearity clusters.

The fluxionality of the bridging hydrides has been revealed by ${ }^{13} \mathrm{C}$ NMR study. In contrast to the crystal structure which possesses no apparent symmetry, the ${ }^{13} \mathrm{C}$ NMR spectrum recorded at room temperature shows only six CO signals at $\delta 242.5\left(J_{\mathrm{P}-\mathrm{C}}=39 \mathrm{~Hz}\right)$, $237.2,199.2,195.5,192.9$ and 192.4 in the ratio $2: 1: 2: 1: 2: 2$ (Fig. 2). The doublet at $\delta 242.5$ is due to the unique bridging $\mathrm{CO}$ ligands, because of its downfield chemical shift and large ${ }^{2} J_{P_{-}-}$coupling with the phosphorus atom which is located at the trans position. The signals at $\delta 237.2$ and 195.5 are assigned to the $\mathrm{Mo}-\mathrm{CO}$ and the axial $\mathrm{CO}$ on atom $\mathrm{Ru}(1)$, respectively. On the basis of these spectral assignments, we deduce that the edge-bridging hydride ligand undergoes rapid migration between the $R u(1)-R u(3)$ and $R u(1)-R u(2)$ bonds, which generates a time-averaged mirror plane and simplifies the pattern of $\mathrm{CO}$ resonances but fails to average the local environment of hydrides in the ${ }^{1} \mathrm{H}$ NMR spectrum at the same temperature. However, irradiation of either hydride signal under the conditions of a NMR spin-saturation transfer experiment suppressed the second hydride signal, suggesting that exchange between edge and triply bridging hydride is feasible at this temperature, but the energy barrier is slightly greater than that for the migration of the edge-bridging hydride ligand. A similar hydride migration between the adjacent $\mathrm{Ru}-\mathrm{Ru}$ bonds was established for the heterometallic cluster $\mathrm{CpWRu}_{3}(\mathrm{CO})_{11^{-}}$ $(\mu-\mathrm{H})_{2}\left(\mathrm{AuPPh}_{3}\right)$ [18], for which exchange between edge- and face-bridging was not detected at temperature below $240 \mathrm{~K}$.
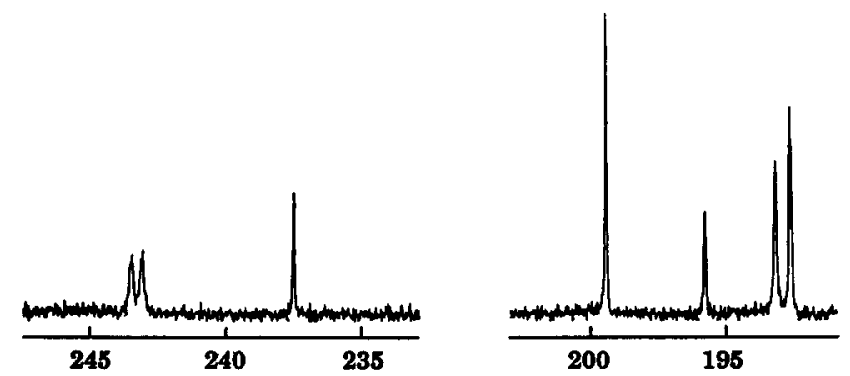

Fig. $2 .{ }^{13} \mathrm{C}-\left\{{ }^{1} \mathrm{H}\right\}$ NMR spectrum $(100.6 \mathrm{MHz})$ of 5 recorded at $294 \mathrm{~K}$, showing the region of CO resonances. 


\subsection{Characterization of 6 and 7}

Complexes 6 and 7 were characterized by spectral methods. The ${ }^{13} \mathrm{C}$ NMR spectrum of 6 shows two bridging $\mathrm{CO}$ resonances at $\delta 261.7$ and $245.0\left(J_{\mathrm{P}-\mathrm{C}}=\right.$ $30 \mathrm{~Hz})$, a Mo-CO resonance at $\delta 222.5\left(J_{\mathrm{P}-\mathrm{C}}=14 \mathrm{~Hz}\right)$ and five $\mathrm{Ru}-\mathrm{CO}$ resonances in the range $\delta$ 198.5-191.9 in the ratio $1: 3: 1: 1: 1$. The ${ }^{1} \mathrm{H}$ NMR spectrum which reveals a signal at $\delta-19.14$ for the hydride, and ${ }^{31} P$ NMR spectrum which exhibits a phosphinidene signal at $\delta 410.7$, which is near the value found for $\mu_{3}$-phosphinidene ligands in $\mathrm{Ru}_{4}$ clusters [19], supplement the information obtained from the ${ }^{13} \mathrm{C} N M R$ spectrum. In addition, the $\nu(\mathrm{CO})$ pattern in solution IR spectrum, featuring seven terminal $\mathrm{CO}$ bands in the range 2063$1893 \mathrm{~cm}^{-1}$ and two bridging $\mathrm{CO}$ stretching bands at 1870 and $1796 \mathrm{~cm}^{-1}$, is fully comparable with that of a structurally characterized $\mathrm{WRu}_{3}$ analogue 2 . These experimental data provide unambiguous proof of the proposed structure.

Likewise, the ${ }^{1} \mathrm{H}$ and ${ }^{31} \mathrm{P}$ NMR spectra of 7 exhibit a hydride signal at $\delta-19.04\left(J_{\mathrm{P}-\mathrm{H}}=14.3 \mathrm{~Hz}\right)$ and a phosphinidene resonance at $\delta 299.9$, respectively; no observation of a second set of hydride and phosphinidene signals was made even at $200 \mathrm{~K}$, suggesting that this molecule contains only one isomer in solution. The ${ }^{13} \mathrm{C}$ NMR spectrum recorded at $213 \mathrm{~K}$, showing four CO signals at $\delta 239.9,197.3\left(J_{\mathrm{P}-\mathrm{C}}=43 \mathrm{~Hz}\right), 192.3$ $\left(J_{\mathrm{P}-\mathrm{C}}=7 \mathrm{~Hz}\right)$ and $192.1\left(J_{\mathrm{P}-\mathrm{C}}=4 \mathrm{~Hz}\right)$ in an intensity ratio $2: 2: 2: 2$, implies that the hydride is located on the unique $\mathrm{Ru}-\mathrm{Ru}$ edge in solution. As verified by this low-temperature ${ }^{13} \mathrm{C}$ NMR work, complex 7 in solution is isostructural with the phosphinidene cluster $1 \mathrm{a}$ and the imido clusters $\mathrm{LWRu}_{2}(\mathrm{CO})_{8}(\mu-\mathrm{H})\left(\mu_{3}-\mathrm{NPh}\right)$. In contrast, a solution of the $\mathrm{Cp}^{\star} \mathrm{WRu}_{2}$ analogue $\mathbf{1 b}$ contains two interconvertible isomers, which are related by rapid hydride migration between $\mathrm{W}-\mathrm{Ru}$ and $\mathbf{R u}-\mathbf{R u}$ edges.

\subsection{Characterization of 8}

Thermolysis of 7 in refluxing toluene solution induced elimination of $\mathrm{H}_{2}$ and caused dimerization to afford hexanuclear 8 in $49 \%$ yield. The ${ }^{13} \mathrm{C}$ NMR

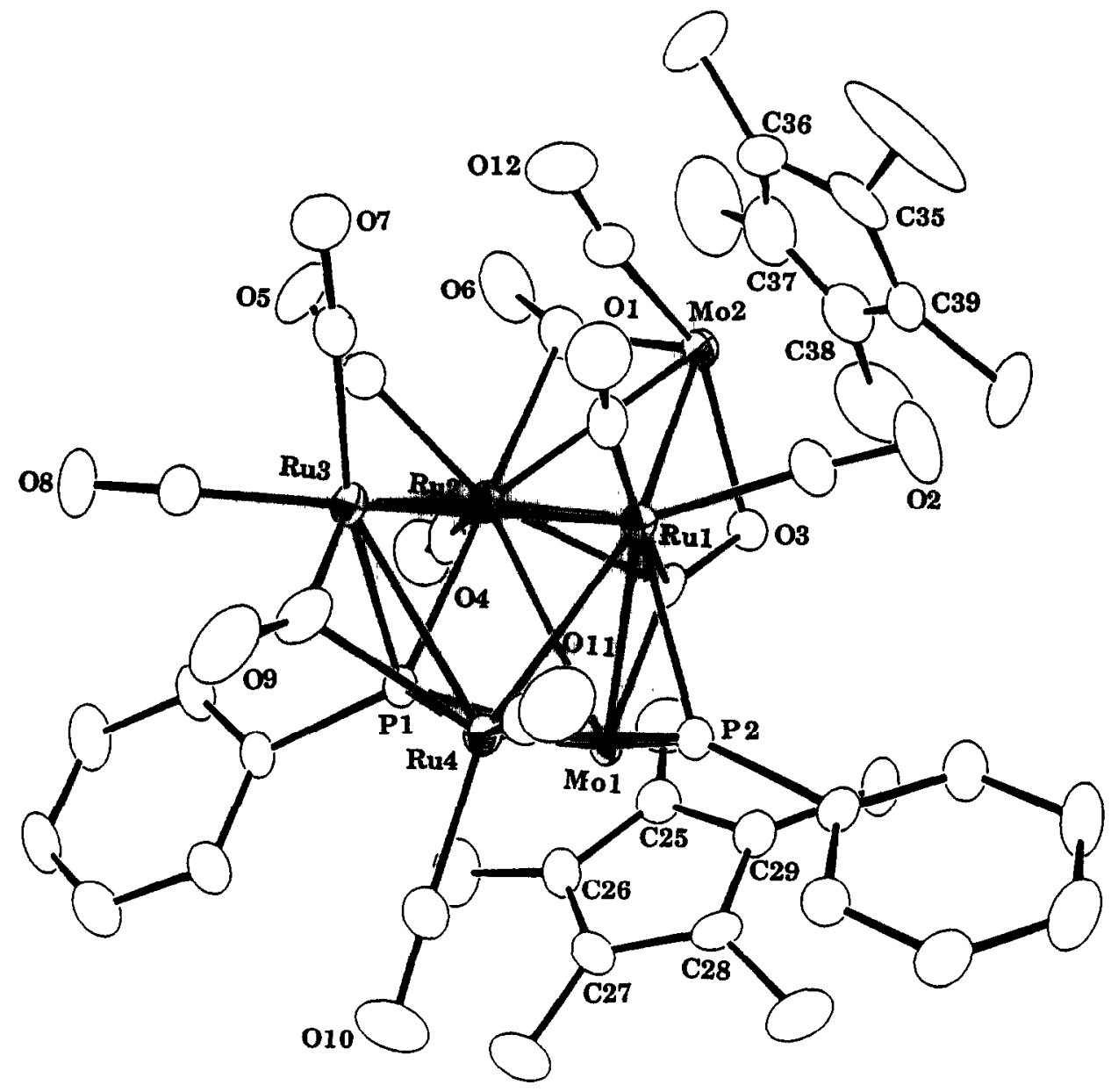

Fig. 3. Molecular structure of 8 and the atomic numbering scheme. 
spectrum recorded at $218 \mathrm{~K}$ shows an $\eta^{2}$-CO signal at $\delta 271.5\left(J_{\mathrm{P}-\mathrm{H}}=8.5 \mathrm{~Hz}\right)$, the bridging $\mathrm{Mo}(\mu-\mathrm{CO}) \mathrm{Ru}$ signal at $\delta 256.0\left(J_{\mathrm{P}-\mathrm{H}}=8 \mathrm{~Hz}\right)$, the bridging $\mathrm{Ru}(\mu$ $\mathrm{CO}) \mathrm{Ru}$ signal at $\delta 235.2\left(J_{\mathrm{P}-\mathrm{H}}=8 \mathrm{~Hz}\right)$, the terminal Mo-CO signal at $\delta 231.0$ and eight terminal $\mathrm{Ru}-\mathrm{CO}$ resonances in the range 217.9-184.3. The low temperature ${ }^{13} \mathrm{C}$ NMR data of the $\mathrm{W}_{2} \mathrm{Ru}_{4}$ analogue 4 reported earlier assists the assignment.

The crystal structure of 8 was also determined by single-crystal $X$-ray diffraction. A molecular drawing appears in Fig. 3 while the essential bond distances and angles are summarized in Table 5. Compound 8 possesses a hexanuclear core of two molybdenum and four ruthenium atoms coordinated by $12 \mathrm{CO}$ ligands and two phosphinidene ligands. The core skeleton and ligand arrangement is virtually the same as that of the related $\mathrm{W}_{2} \mathrm{Ru}_{4}$ cluster 4 , comprising an edge-bridged square pyramidal $\mathrm{MoRu}_{4}$ central core, a tentacle Mo metal unit, two phosphinidene ligands, and a $\mu_{4}-\eta^{2}-\mathrm{CO}$ ligand encapsulated in the cavity of the $\mathrm{Mo}_{2} \mathrm{Ru}_{2}$ butterfly. In this molecule all metal-metal bonds are normal with $\mathrm{Ru}-\mathrm{Ru}$ distances in the range $2.770(1)$ 3.037(1) $\AA$ and $\mathrm{Mo}-\mathrm{Ru}$ distances in the narrow range 2.904(1)-3.006(1) $\AA$; one $\mu_{3}$-phosphinidene ligand occupies a $\mathrm{MoRu}_{2}$ metal triangle and a second $\mu_{4}$-phosphinidene takes the $\mathrm{MoRu}_{3}$ square face. If we consider that the $\mu_{4}$-phosphinidene ligands are part of the cluster core, the molecule appears to adopt a $\mathrm{MoRu}_{4} \mathrm{P}$ octahedral core bridged by a $\mu_{3}$-phosphinidene and a $\mathrm{Cp}^{\star} \mathrm{Mo}(\mathrm{CO})_{2}$ fragment. A few cluster complexes containing both $\mu_{4^{-}}$and $\mu_{3}$-phosphinidene ligands [20] or clusters with the unique $\mu_{4}-\eta^{2}-\mathrm{CO}$ ligand [21] have been documented.

\section{Summary}

Reactions performed in this work are summarized in Scheme 2. Condensation of $\mathrm{Ru}_{3}(\mathrm{CO})_{10}(\mu-\mathrm{H})(\mu-$ $\mathrm{PPh}_{2}$ ) and $\mathrm{Cp}^{\star} \mathrm{Mo}(\mathrm{CO})_{3} \mathrm{H}$ proved to be a convenient method to generate the $\mathrm{MoRu}_{3}$ phosphido cluster 5 . Its formation is best interpreted as a cluster condensation, in which the reaction proceeded via formation of unsaturated $\mathrm{Ru}_{3}(\mathrm{CO})_{9}(\mu-\mathrm{H})\left(\mu-\mathrm{PPh}_{2}\right)$, followed by addition of one molecule of $\mathrm{Cp}^{*} \mathrm{Mo}(\mathrm{CO})_{3} \mathrm{H}$. Thermolysis of 5 in toluene produced the phosphinidene cluster 6 in moderate yield via elimination of a benzene molecule. Isolation of 5 provides unambiguous evidence that, for the $\mathrm{MoRu}_{3}$ phosphinidene cluster 6, the formation of tetrahedral cluster core occurred prior to the generation of phosphinidene ligand by elimination of benzene. In contrast, Mays and coworkers reported that the tetrahedral phosphinidene cluster complexes can be prepared by condensation of anionic phosphinidene cluster $\left[\mathrm{Ru}_{3}(\mathrm{CO})_{9}(\mu-\mathrm{H})\left(\mu_{3}-\mathrm{PPh}\right)\right]^{-}$with a cationic, mononuclear metal fragment [22].

Furthermore, complex 6 reacted with $\mathrm{CO}$ to afford the trinuclear phosphinidene cluster 7 through cluster fragmentation. In contrast to the $\mathrm{WRu}_{2}$ counterpart, it exists in solution as only one isomer, in which the hydride ligand is associated with the unique $R u-R u$ edge [3]. The variation of isomer proportion indicates that molybdenum donates fewer electrons than tungsten, thus disfavouring the move of the hydride from the $\mathbf{R u}-\mathbf{R u}$ to the Mo-Ru edge. Similar behaviour has also been noted upon replacing the $\mathrm{Cp}^{\star}$ ligand with the less markedly electron-donating $\mathrm{Cp}$ ligand in the $\mathrm{WRu}_{2}$ system [3]. When complex 7 was heated in
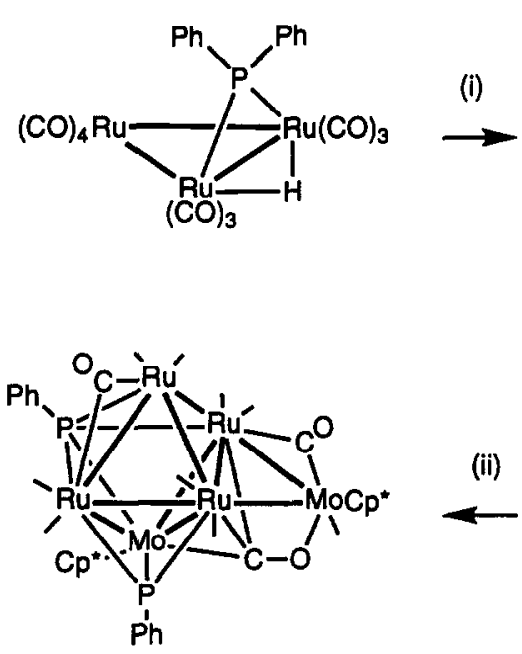

(8)

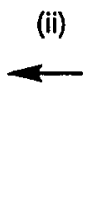

Scheme 2.

(7)

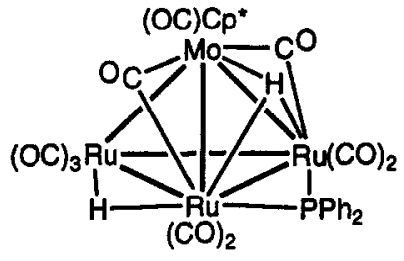

(5)
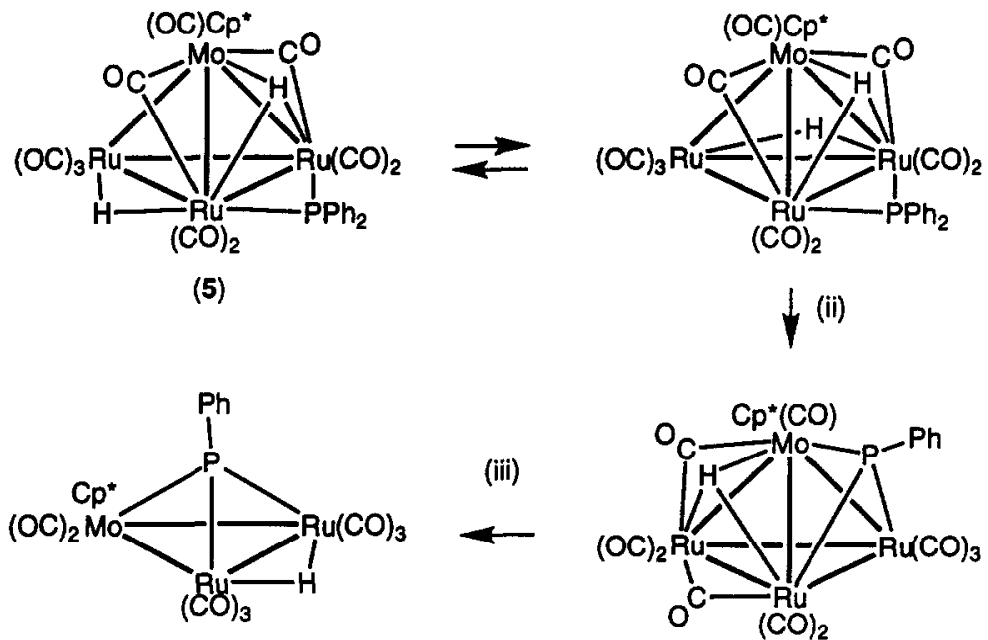

(iii) $\downarrow$ (ii)

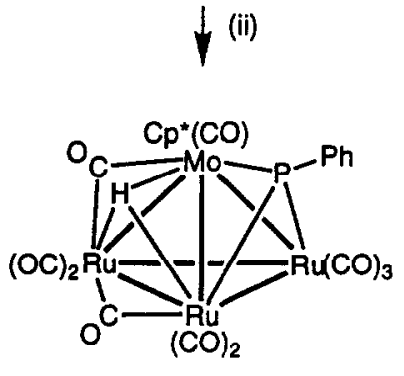

(6) 
refluxing toluene, the hexanuclear cluster 8 was obtained. Interestingly, complex 8 remains the only $\mathrm{Mo}_{2} \mathrm{Ru}_{4}$ cluster observed in solution upon extending the reaction. This fruitless attempt indicates that the $\mathrm{Mo}_{2} \mathrm{Ru}_{4}$ analogue of hexanuclear $\mathrm{W}_{2} \mathrm{Ru}_{4}$ cluster 3 is probably absent. The factors controlling the geometry of the cluster skeleton remains a goal of further research.

\section{Acknowledgment}

We thank the National Science Council of the Republic of China for financial support (Grant No. NSC83-0208-M007-43).

\section{References}

1 (a) D.A. Roberts and G.L. Geoffroy, in G. Wilkinson, F.G.A. Stone and E.W. Abel (eds.), Comprehensive Organometallic Chemistry Pergamon, Oxford, 1982, Vol. 6, Chapter 40; (b) D.F. Shriver, H.D. Kaesz and R.D. Adams (eds.), The Chemistry of Metal Cluster Complexes, VCH Publishers, New York, 1990.

2 (a) Y. Chi, L.-K. Liu, G. Huttner and L. Zsolnai, J. Organomet. Chem., 390 (1990) C50; (b) Y. Chi, H.-F. Hsu, L.-K. Liu, S.-M. Peng and G.-H. Lee, Organometallics, $1 /$ (1992) 1763.

3 R.-C. Lin, Y. Chi, S.-M. Peng and G.-H. Lee, Inorg. Chem., 31 (1992) 3818

4 (a) Y. Chi, R.-C. Lin, S.-H. Peng and G.-H. Lee, J. Cluster Sci., 3 (1992) 333; (b) R.-C. Lin, Y. Chi, S.-H. Peng and G.-H. Lee, J. Chem. Soc., Dalton Trans., (1993) 227; (c) R.-C. Lin, Y. Chi, S.-H. Peng and G.-H. Lee, J. Chem. Soc., Chem. Commun., (1992) 1705.

5 J.-C. Wang, R.-C. Lin, Y. Chi, S.-H. Peng and G.-H. Lee, Organometallics, 12 (1993) 4061.

6 C.E. Housecroft, in T.P. Fehlner (ed.), Inorganometallic Chemistry, Plenum Press, New York, 1992, Chapter 3.

7 S.P. Rowley, P.S. White and C.K. Schauer, Inorg. Chem., 31 (1992) 3158.

8 M. Lanfranchi, A. Tiripicchio, E. Sappa and A.J. Carty, J. Chem. Soc., Dalton Trans., (1986) 2737.
9 D. Nucciarone, S.A. MacLaughlin and A.J. Carty, Inorg. Synth. 26 (1989) 264.

10 S.P. Nolan and C.D. Hoff, in R.B. King and J.J. Eisch (eds.), Organometallic Syntheses, Elsevier, New York, 1989, Vol. 4, p. 58.

11 S.A. MacLaughlin, A.J. Carty and N.J. Taylor, Can. J. Chem., 60 (1982) 87.

12 K. Natarajan, L. Zsolnai and G. Huttner, J. Organomet. Chem., 209 (1981) 85.

13 J.S. Field, R.J. Haines and D.N. Smit, J. Organomet. Chem., 224 (1982) C49.

14 A.P. Humphries and H.D. Kaesz, Prog. Inorg. Chem., 25 (1979) 146.

15 (a) S.A. MacLaughlin, N.J. Taylor and A.J. Carty, Organometallics, 2 (1983) 1194; (b) M.I. Bruce, M.R. Snow, E.R.T. Tiekink and M.L. Williams, J. Chem. Soc., Chem. Commun., (1986) 701; (c) M.I. Bruce, M.J. Liddell, B.W. Skelton and A.H. White, Organometallics, 10 (1991) 3282; (d) J.S. Field, R.J. Haines and F. Mulla, J. Organomet. Chem., 439 (1992) C56.

16 M. Green, K.A. Mead, R. Mills, I.D. Salter, F.G.A. Stone and P. Woodward, J. Chem. Soc., Chem. Commun., (1982) 51.

17 B.T. Huie, C.B. Knobler and H.D. Kaesz, J. Am. Chem. Soc., 100 (1978) 3059

18 C.-C. Chen, Y. Chi, S.-H. Peng and G.-H. Lee, J. Chem. Soc., Dalton Trans., (1993) 1823.

19 F. Van Gastel, J.F. Corrigan, S. Doherty, N.J. Taylor and A.J. Carty, Inorg. Chem., 31 (1992) 4492.

20 (a) K. Kwek, N.J. Taylor and A.J. Carty, J. Am. Chem. Soc., 106 (1984) 4636; (b) J.S. Field, R.J. Haines and F. Mulla, J. Organomet. Chem., 389 (1990) 227.

21 (a) M. Manassero, M. Sansoni and G. Longoni, J. Chem. Soc., Chem. Commun., (1976) 919; (b) B.F.G. Johnson, J. Lewis, M. McPartlin, M. Pearsall and A. Sironi, J. Chem. Soc., Chem. Commun., (1984) 1089; (c) C.P. Horwitz and D.F. Shriver, Organometallics, 3 (1984) 756; (d) C.P. Horwitz and D.F. Shriver, J. Am. Chem. Soc., 107 (1985) 8147; (e) C.P. Horwitz, E.M. Holt, C.P. Brock and D.F. Shriver, J. Am. Chem. Soc., 107 (1985) 8136; (f) R.D. Adams, J.E. Babin and M. Tasi, Inorg. Chem., 27 (1988) 2618; (g) C.E. Anson, P.J. Bailey, G. Conole, B.F.G. Johnson, J. Lewis, M. McPartlin and H.R. Powell, J. Chem. Soc., Chem. Commun., (1989) 442; (h) Y. Chi, F.-J. Wu, B.-J. Liu, C.-C. Wang and S.-L. Wang, J. Chem. Soc., Chem. Commun., (1989) 873; (i) Y. Chi, S.-H. Chuang, L.-K. Liu and Y.-S. Wen, Organometallics, 10 (1991) 2485.

22 M.J. Mays, P.R. Raithby, P.L. Taylor and K. Henrick, J. Chem. Soc., Dalton Trans., (1984) 959. 